University of Nebraska - Lincoln

DigitalCommons@University of Nebraska - Lincoln

$11-2003$

\title{
Screw dislocation interacting with twin interfacial edge cracks between two bonded dissimilar piezoelectric strips
}

Xiangfa Wu

Department of Engineering Mechanics, University of Nebraska-Lincoln, xfwu@unlserve.unl.edu

Yuris A. Dzenis

Department of Engineering Mechanics,University of Nebraska-Lincoln, ydzenis@unl.edu

Tian-You Fan

Beijing Institute of Technology, China

Follow this and additional works at: https://digitalcommons.unl.edu/engineeringmechanicsfacpub

Part of the Mechanical Engineering Commons

Wu, Xiangfa; Dzenis, Yuris A.; and Fan, Tian-You, "Screw dislocation interacting with twin interfacial edge cracks between two bonded dissimilar piezoelectric strips" (2003). Faculty Publications from the Department of Engineering Mechanics. 30.

https://digitalcommons.unl.edu/engineeringmechanicsfacpub/30

This Article is brought to you for free and open access by the Mechanical \& Materials Engineering, Department of at DigitalCommons@University of Nebraska - Lincoln. It has been accepted for inclusion in Faculty Publications from the Department of Engineering Mechanics by an authorized administrator of DigitalCommons@University of Nebraska - Lincoln. 
Published in Mechanics Research Communications 30:6 (November-December 2003), pp. 547-555; doi 10.1016/S0093-6413(03)00062-4 Copyright (C) 2003 Elsevier Ltd. Used by permission. http://www.elsevier.com/wps/product/cws_home/374

Submitted December 27, 2002; published online June 26, 2003.

\title{
Screw dislocation interacting with twin interfacial edge cracks between two bonded dissimilar piezoelectric strips
}

\author{
Xiang-Fa Wu, ${ }^{1}$ Yuris A. Dzenis, ${ }^{1}$ and Tian-You Fan ${ }^{2}$ \\ 1 Department of Engineering Mechanics, Center for Materials Research and Analysis, \\ University of Nebraska-Lincoln, Lincoln, NE 68588-0526, USA \\ 2 Department of Applied Physics, College of Science, \\ Beijing Institute of Technology, Beijing 100081, China \\ Corresponding author - X.-F. Wu
}

\begin{abstract}
This paper is concerned with the electroelastic potentials and the fracture parameters of a twinedge-cracked piezoelectric bimaterial strip with a screw dislocation. By means of conformal mapping technique and the known dislocation solution, the antiplane displacement and inplane electric potentials are obtained in closed-form. The intensity factors and the energy release rate are extracted explicitly. In some limiting cases, the present solutions cover those in the literature.
\end{abstract}

\section{Introduction}

Due to their intrinsic electromechanical coupling property, piezoelectric materials are used extensively in electromechanical devices such as actuators, sensors, and transducers, etc. Piezoelectric materials such as ferroelectric ceramics are brittle and liable to cracking at the multitude of scales, from domains to devices. Under external electromechanical loadings, cracks and delaminations may initiate and grow from microdefects such as dislocations, microcracks, cavities, and inclusions, etc. Theoretically, dislocation solutions play an important role in the study of the behaviors of various defects in piezoelectric materials under coupled electromechanical loadings. Since the pioneering works by Parton (1976) and Deeg (1980), a significant progress has been made on the electroelastic fields of piezoelectric materials disturbed by dislocations, cracks, cavities, and inclusions, for example, Suo et al. (1992) discussed the general solutions for collinear interfacial cracks in anisotropic piezoelectric bimaterials. Recent development in fracture mechanics of piezoelectric materials can be found in the review paper by Zhang et al. (2002).

In this work we pay attention to the explicit solutions of screw dislocations in edge-cracked piezoelectric bimaterial strips. Screw dislocation in piezoelectric materials is one of the simplest while 
most important dislocation cases discussed extensively by a number of researchers in the last decade. To mention a few, Pak (1990) first introduced the Peach-Koehler forces acting on a screw dislocation in an infinite piezoelectric medium. Using complex analysis and conformal mapping, $\mathrm{Li}$ and Duan (2001) considered a screw dislocation in a homogenous piezoelectric cylinder and strip. In the cases of dislocation/crack interactions, Lee et al. (2000), Chen et al. (2002a), and Kwon and Lee (2002) studied the electroelastic fields and the fracture parameters of finite and semi-infinite cracks in transversely homogenous piezoelectric ceramics involving a screw dislocation. In the bimaterial cases, Liu et al. (1999) obtained the electroelastic potential solutions for a screw dislocation located in an infinite bimaterial medium. Furthermore, by means of dual integral equations, Li and Fan (2001) analyzed an interfacial edge crack in a piezoelectric bimaterial half-plane. Soh et al. (2002) and Wu et al. (2003) obtained the general solutions for a screw dislocation interacting with an interfacial crack in piezoelectric bimaterials by means of perturbation theory. Moreover, using complex analysis, Chen et al. (2002b) and Wu et al. (2002) further concerned a screw dislocation in a piezoelectric bimaterial wedge. In practice, piezoelectric devices are made of piezoelectric laminas. As a fact, it is necessary to investigate the electroelastic fields and the fracture parameters of cracked piezoelectric laminates in order to explore their failure mechanisms and potential structure optimization. Recently, Li (2002) and Li and Tang (2002) discussed the electrically permeable cracks and edge cracks in piezoelectric strips, while general solutions for these edge-cracked piezoelectric strips are lacking. In the present work, a screw dislocation in a twin-edge-cracked piezoelectric bimaterial strip is concerned. By means of conformal mapping technique and the known solution of a screw dislocation in a piezoelectric bimaterial (Liu et al., 1999; Wu et al., 2003), the antiplane displacement and inplane electric potentials of the discussed piezoelectric strip are obtained in closed-form. The intensity factors (IFs) and the energy release rates (ERRs) of the edge cracks are extracted explicitly.

\section{Problem statement and solution procedure}

As shown in Figure 1, assume the piezoelectric materials transversely isotropic with an isotropic basal plane parallel to $x y$-plane and a poling direction perpendicular to $x y$-plane (Deeg, 1980; Pak, 1990). The piezoelectric boundary-value problem is treated as in the case of antiplane mechanical displacement and inplane electric fields such that

$$
\begin{aligned}
& u_{x}=u_{y}=0, \quad u_{z}=u_{z}(x, y), \\
& E_{x}=E_{x}(x, y), \quad E_{y}=E_{y}(x, y), \quad E_{z}=0 .
\end{aligned}
$$

Thus, the constitutive relations reduce to

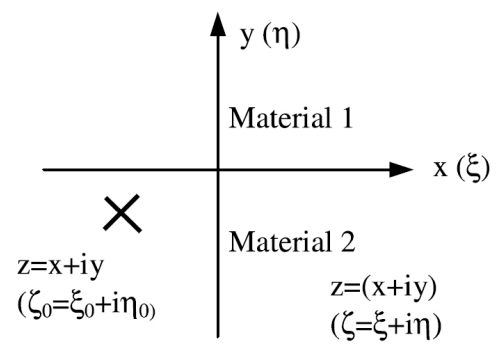

Figure 1. Screw dislocation in a piezoelectric bimaterial. 


$$
\begin{array}{ll}
\sigma_{x z}=c_{44} u_{z, x}+e_{15} \phi_{, x^{\prime}} & D_{x}=e_{15} u_{z, x}-\varepsilon_{11} \phi_{, x^{\prime}} \\
\sigma_{y z}=c_{44} u_{z, y}+e_{15} \phi_{, y^{\prime}} & D_{y}=e_{15} u_{z, y}-\varepsilon_{11} \phi_{, y^{\prime}}
\end{array}
$$

where $\sigma_{k z^{\prime}} D_{k}(k=x, y), c_{44^{\prime}} e_{15}, \varepsilon_{11}$ and $\phi$ are the stress tensor, electric displacement vector, elastic modulus at constant electric field, piezoelectric constants, and electric potential, respectively.

The electric field is given by

$$
E_{x}=-\phi_{, x^{\prime}} \quad E_{y}=-\phi_{, y^{\prime}}
$$

and the piezoelectric governing equations are

$$
\sigma_{x z, x}+\sigma_{y z, y}=0, \quad D_{x, x}+D_{y, y}=0 .
$$

Equations (5) may be rewritten in terms of $\phi$ and $u_{z}$ as

$$
c_{44} \nabla^{2} u_{z}+e_{15} \nabla^{2} \phi=0, \quad e_{15} \nabla^{2} u_{z}-\varepsilon_{11} \nabla^{2} \phi=0,
$$

and can be further reduced to

$$
\nabla^{2} u_{z}=0, \quad \nabla^{2} \phi=0 .
$$

As a result, the antiplane displacement $u_{z}$ and the electric potential $\phi$ satisfy two decoupled Laplace equations, and the solutions can be expressed as the imaginary parts of two analytic functions $U(z)$ and $\Phi(z)$ such that

$$
u_{z}=\operatorname{Im}[U(z)], \phi=\operatorname{Im}[\Phi(z)]
$$

where $\operatorname{Im}($ ) denotes the imaginary part of an analytic function.

It is convenient to introduce the complex stress and the electric displacement such that

$$
\begin{aligned}
& \sigma_{z y}+\mathrm{i} \sigma_{z x}=c_{44}\left(u_{z, y}+\mathrm{i} u_{z, x}\right)+e_{15}\left(\phi_{, y}+\mathrm{i} \phi_{, x}\right), \\
& D_{y}+\mathrm{i} D_{x}=e_{15}\left(u_{z, y}+\mathrm{i} u_{z, x}\right)-\varepsilon_{11}\left(\phi_{, y}+\mathrm{i} \phi_{, x}\right) .
\end{aligned}
$$

Substitution of (8) into (9) leads to

$$
\begin{gathered}
\sigma_{z y}+\mathrm{i} \sigma_{z x}=c_{44} U^{\prime}(z)+e_{15} \Phi^{\prime}(z), \\
D_{y}+\mathrm{i} D_{x}=e_{15} U^{\prime}(z)-\varepsilon_{11} \Phi^{\prime}(z) .
\end{gathered}
$$

\subsection{Screw dislocation in a piezoelectric bimaterial (Liu et al., 1999; Wu et al., 2003)}

The solution of this problem has been given by Liu et al. (1999) and Wu et al. (2003) using complex variables. Since it is fundamental to the current study, we briefly describe the process. Here we further reserve the $x y$-coordinate system for the problem to be solved. Without loss of generality, as shown in Figure 1, suppose the screw dislocation is located in the lower half-plane at $\zeta_{0}\left(\zeta_{0}=\xi_{0}+\mathrm{i} \eta_{0^{\prime}}\right.$ $\eta_{0}<0$ ) and is characterized by Burgers vector $b$, line-force $p$, line-charge $q$, and electric potential jump $\Delta \phi$. The electroelastic potentials can be expressed as

$$
U(\zeta)= \begin{cases}U_{1}(\zeta), & \zeta \in D_{1} \text { i.e. }(\eta>0), \\ U_{2}(\zeta)+U_{0}(\zeta), & \zeta \in D_{2} \text { i.e. }(\eta<0)\end{cases}
$$

and

$$
\Phi(\zeta)= \begin{cases}\Phi_{1}(\zeta), & \zeta \in D_{1} \text { i.e. }(\eta>0) \\ \Phi_{2}(\zeta)+\Phi_{0}(\zeta), & \zeta \in D_{2} \text { i.e. }(\eta<0)\end{cases}
$$


Here $U_{1}(z), U_{2}(z), \Phi_{1}(z)$ and $\Phi_{2}(z)$ are unknown electroelastic potentials to be determined, and $U_{0}(z)$ and $\Phi_{0}(z)$ are electroelastic potentials for the screw dislocation in an infinite homogenous piezoelectric material expressed as follows (Pak, 1990)

$$
\left[\begin{array}{l}
U_{0}(\zeta) \\
\Phi_{0}(\zeta)
\end{array}\right]=\mathbf{T} \ln \left(\zeta-\zeta_{0}\right)
$$

where

$$
\mathbf{T}=\frac{1}{2 \pi}\left[\begin{array}{c}
b \\
\Delta \phi
\end{array}\right]+\frac{L^{-1}}{2 \pi \mathrm{i}}\left[\begin{array}{c}
p \\
-q
\end{array}\right], \quad L_{2}=\left[\begin{array}{cc}
c_{44} & e_{15} \\
e_{15} & -\varepsilon_{11}
\end{array}\right] .
$$

The above elements in material matrix $L_{2}$ are referring to the lower half-plane.

In order to simplify the derivation, we introduce five vectors

$$
\mathbf{f}_{1}(\zeta)=\left[\begin{array}{l}
U_{1}(\zeta) \\
\Phi_{1}(\zeta)
\end{array}\right], \quad \mathbf{f}_{2}(\zeta)=\left[\begin{array}{l}
U_{2}(\zeta) \\
\Phi_{2}(\zeta)
\end{array}\right], \quad \mathbf{f}_{0}(\zeta)=\left[\begin{array}{l}
U_{0}(\zeta) \\
\Phi_{0}(\zeta)
\end{array}\right],
$$

and

$$
\mathbf{u}=\left[\begin{array}{c}
u_{z} \\
\phi
\end{array}\right], \quad \mathbf{t}=\left[\begin{array}{c}
\sigma_{y z} \\
D_{y}
\end{array}\right]=L_{j} / 2\left[\mathbf{f}^{\prime}(\zeta)+\overline{\mathbf{f}}^{\prime}(\zeta)\right]
$$

Thus, the solution for this problem can be expressed as follows (Liu et al., 1999; Wu et al., 2003)

$$
\begin{aligned}
& \mathbf{f}_{1}(\zeta)=2\left(L_{1}+L_{2}\right)^{-1} L_{2} \mathbf{f}_{0}(\zeta), \quad \zeta \in D_{1}, \\
& \mathbf{f}_{2}(\zeta)=\left(L_{1}+L_{2}\right)^{-1}\left(L_{1}-L_{2}\right) \overline{\mathbf{f}}_{0}(\zeta), \quad \zeta \in D_{2} .
\end{aligned}
$$

where $L_{1}$ and $L_{2}$ stand for the material matrices of the half-planes above and below defined in (14).

\subsection{Screw dislocation in a piezoelectric bimaterial strip}

Consider a screw dislocation located in a piezoelectric bimaterial strip with width $W$, as shown in Figure 2(a). Assume the dislocation located in the lower strip at $z_{0}=x_{0}+\mathrm{i} y_{0}\left(y_{0}<0\right)$, $x$ and $y$ being the coordinates of the material points, and the strip surfaces being stress-free and electrically

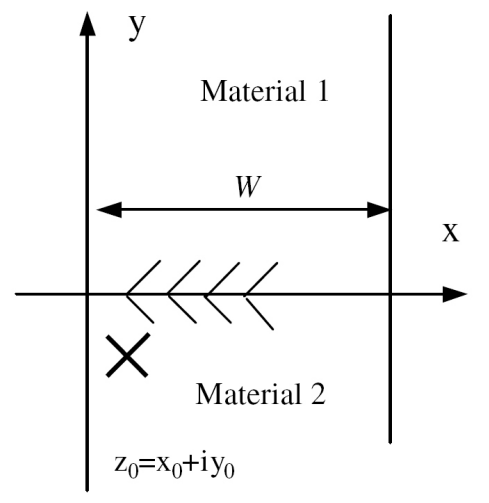

(a)

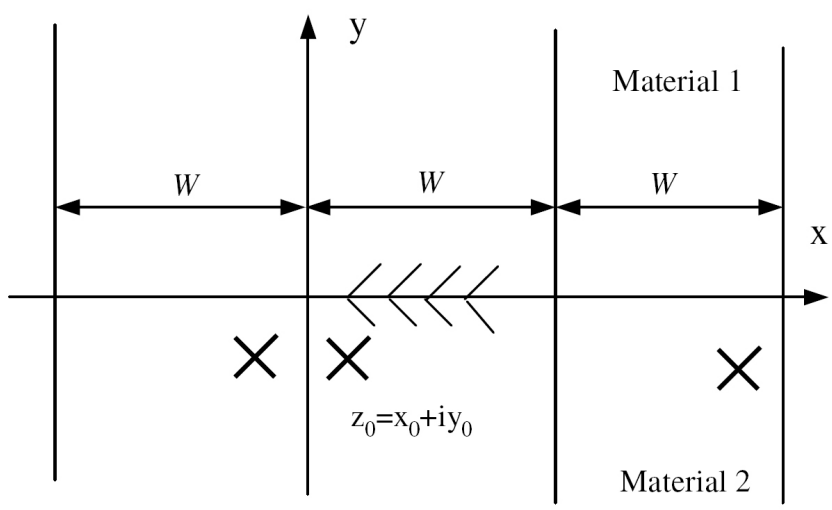

(b)

Figure 2. Piezoelectric bimaterial with screw dislocations: (a) two bonded strips (z-plane), (b) two bonded halfplanes with periodic screw dislocations (z-plane). 
impermeable. This problem can be solved as a periodic screw dislocation problem, as shown in Figure 2(b), where screw dislocations with equal quantities $(b, \Delta \Phi, p, q)$ are located periodically at $z_{n}=$ $\left(2 N W+x_{0}\right)+\mathrm{i} y_{0}$ and $z_{-n}=\left(2 N W-x_{0}\right)+\mathrm{i} y_{0}(N=0, \pm 1, \pm 2 \ldots \pm \infty)$. The typical strip element for this periodic problem is shown in Figure 3(a), in which two screw dislocations with $(b, \Delta \Phi, p, q)$ are located symmetrically at $z_{1}=x_{0}+\mathrm{i} y_{0}$ and $z_{2}=\left(2 W-x_{0}\right)+\mathrm{i} y_{0}$ in two bonded dissimilar piezoelectric strips with width $2 W$.

Since the displacement and electric potentials in each piezoelectric material are holomorphic, the potential solutions for this strip element can be constructed using conformal mapping technique. Introduce the conformal mapping

$$
\zeta=\operatorname{tg} \frac{\pi(z-W)}{2 W} \quad(z=x+\mathrm{i} y)
$$

which maps the strip element in the z-plane, as shown in Figure 3(a), onto two bond half-planes with two cuts $(\infty,-i)$ and $(i, \infty)$ at the imaginary $\eta$-axis, and the screw dislocations $z_{1}$ and $z_{2}$ are mapped onto

$$
\zeta_{10}=-\operatorname{ctg} \frac{\pi z_{0}}{2 W}, \quad \zeta_{20}=\operatorname{ctg} \frac{\pi \bar{z}_{0}}{2 W}
$$

Due to the periodic property in the physical z-plane, the physical quantities at two sides of the cuts $(\infty,-i)$ and $(i, \infty)$ in the image $\zeta$-plane bear the same values, so we can utilize the dislocation solutions (13) to construct this solution as

$$
\mathbf{f}_{0}(z)=\mathbf{T}\left[\ln \left(\operatorname{ctg} \frac{\pi z_{0}}{2 W}-\operatorname{ctg} \frac{\pi z}{2 W}\right)+\ln \left(-\operatorname{ctg} \frac{\pi \bar{z}_{0}}{2 W}-\operatorname{ctg} \frac{\pi z}{2 W}\right)\right],
$$

where material constants in $\mathrm{T}$ are related to the lower strip.

Substitution of (20) into (17) leads to the potential solutions in the piezoelectric bimaterial strip. With the aid of (10)-(12) and (15)-(16), solutions (17) and (20) can determine the whole electroelastic fields in the strips.

\subsection{Screw dislocation in an edge-cracked piezoelectric strip}

Consider a screw dislocation located in a twin-edge-cracked piezoelectric bimaterial strip as shown in Figure $4(\mathrm{a})$, in which $W, a, b$, and $z_{0}\left(z_{0}=x_{0}+\mathrm{i} y_{0}, y_{0}<0\right)$ denote the strip width, two crack tips, and the location of the screw dislocation, respectively. As discussed in Section 2.2, we solve this problem using solutions (17) and (20) and conformal mapping technique. Here we introduce the conformal mapping

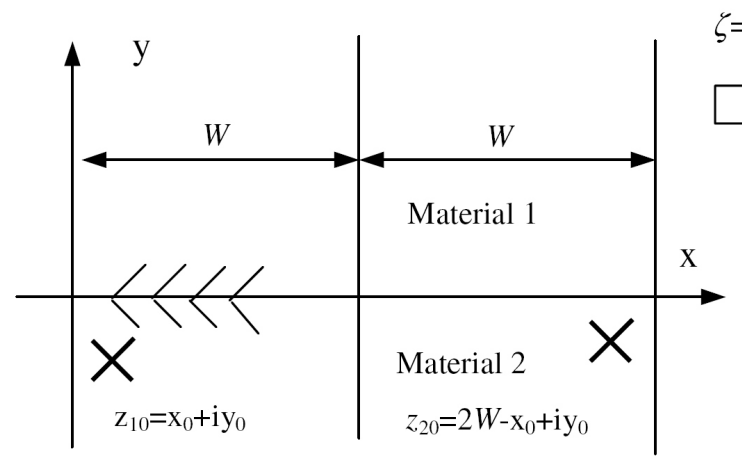

(a)

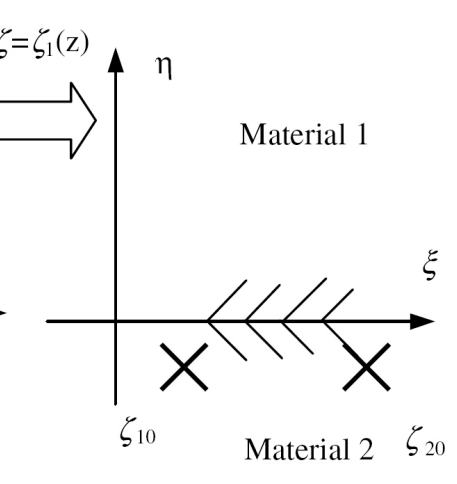

(b)

Figure 3. Piezoelectric bimaterial with screw dislocations: (a) two bonded strips (z-plane), (b) two bonded halfplanes ( $\zeta$-plane). 

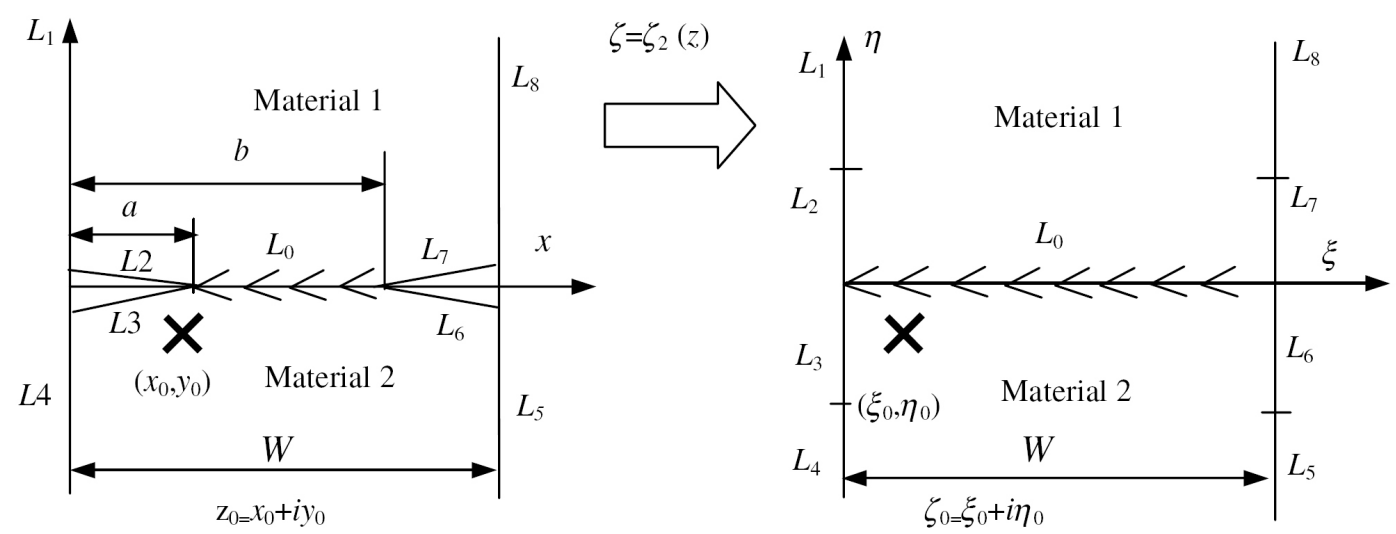

Figure 4. Piezoelectric bimaterial strip with screw dislocation: (a) two bonded strips with twin interfacial edge cracks (z-plane), (b) two bonded strips ( $\zeta$-plane).

$$
\zeta=\frac{2 W}{\pi} \tan ^{-1}\left[\left(\sin ^{2} \frac{\pi z}{2 W}-\sin ^{2} \frac{\pi a}{2 W}\right) /\left(\sin ^{2} \frac{\pi b}{2 W}-\sin ^{2} \frac{\pi z}{2 W}\right)\right]^{1 / 2} \quad(z=x+\mathrm{i} y)
$$

which maps the twin-edge-cracked strip, as shown in Figure 4(a), onto a strip, as shown in Figure $4(\mathrm{~b})$. The screw dislocation located at $z_{0}=x_{0}+\mathrm{i} y_{0}$ in the $z$-plane is mapped onto

$$
\zeta_{0}=\frac{2 W}{\pi} \tan ^{-1}\left[\left(\sin ^{2} \frac{\pi z_{0}}{2 W}-\sin ^{2} \frac{\pi a}{2 W}\right) /\left(\sin ^{2} \frac{\pi b}{2 W}-\sin ^{2} \frac{\pi z_{0}}{2 W}\right)\right],
$$

in the mapped strip ( $\zeta$-plane).

With the help of solution (20), we have

$$
\mathbf{f}_{0}(z)=\mathbf{T}\left\{\ln \left[X\left(z_{0}\right)-X(z)\right]+\ln \left[-\overline{X\left(z_{0}\right)}-X(z)\right]\right\},
$$

where

$$
X(z)=\left[\left(\sin ^{2} \frac{\pi b}{2 W}-\sin ^{2} \frac{\pi z}{2 W}\right) /\left(\sin ^{2} \frac{\pi z}{2 W}-\sin ^{2} \frac{\pi a}{2 W}\right)\right]^{1 / 2},
$$

and $\mathbf{T}$ is defined in (14) with material constants referring to the lower strip.

Substitution of (23) and (24) into (17) yields the general electroelastic potentials of the edgecracked bimaterial strip.

Within the framework of linear fracture mechanics, the most important parameters are the IFs and ERRs of the cracked piezoelectric bodies. In the $x y$-coordinate system as shown in Figure 4(a), the IFs in the left crack tip are defined as

$$
\mathbf{K}=\sqrt{2 \pi} \lim _{x \rightarrow a^{+}}(x-a)^{1 / 2} \mathbf{t}(x)=\sqrt{2 \pi} \lim _{x \rightarrow a^{+}}(x-a)^{1 / 2} L_{1} / 2\left[\mathbf{f}_{\mathbf{1}}^{\prime}(x)+\overline{\mathbf{f}}_{1}^{\prime}(x)\right],
$$

where $\mathbf{K}=\left\{K_{\mathrm{III}}, K_{\mathrm{D}}\right\}^{\mathrm{T}}, K_{\mathrm{D}}$ is the electric IF.

The traction and electric displacement at the interface a distance $r$ ahead of the crack tip and the displacement and electric potential jumps a distance $r$ behind of the crack tip can be expressed as

$$
\mathbf{t}(r)=\mathbf{K} / \sqrt{2 \pi r}, \quad \mathbf{d}(r)=H \mathbf{K} / \sqrt{2 \pi r},
$$


where

$$
\mathbf{d}=\left[u_{z}(x, 0+), \phi(x, 0+)\right]^{\mathrm{T}}-\left[u_{z}\left(x, 0^{-}\right), \phi\left(x, 0^{-}\right)\right]^{\mathrm{T}}, H=\left(L_{1}{ }^{-1}+L_{2}{ }^{-1}\right),
$$

and $H$ is the bimaterial matrix of the piezoelectric bimaterial.

The ERR for a unit crack growth along the interface can be evaluated (Suo et al., 1992) as

$$
G=1 /(2 \Delta) \int_{0}^{\Delta} \mathbf{t}^{\mathrm{T}}(\Delta-r) \cdot \mathbf{d}(r) \mathrm{d} r=(1 / 4) \mathbf{K}^{\mathrm{T}} H \mathbf{K}
$$

In the current problem, substitution of (23) and (24) into the first formula in (17) then into (25) leads to the IFs at $a$ and $b$ as

$$
\begin{aligned}
\mathbf{K}^{a} & =\lim _{x \rightarrow a^{+}} \sqrt{2 \pi(x-a)} L_{1} / 2\left[\mathbf{f}_{1}^{\prime}(x)+\overline{\mathbf{f}_{1}^{\prime}(x)}\right] \\
& =\frac{2 \pi H^{-1}}{\sqrt{\pi a}} \sqrt{\frac{\frac{\pi a}{W} \sin \frac{\pi a}{W}}{\sin ^{2} \frac{\pi b}{2 W}-\sin ^{2} \frac{\pi a}{2 W}}} \operatorname{Re}\left\{-\mathbf{T} \sqrt{\left.\frac{\sin ^{2} \frac{\pi b}{2 W}-\sin ^{2} \frac{\pi z_{0}}{2 W}}{\sin ^{2} \frac{\pi z_{0}}{2 W}-\sin ^{2} \frac{\pi a}{2 W}}\right\},},\right.
\end{aligned}
$$

and

$$
\begin{aligned}
\mathbf{K}^{b} & =\lim _{x \rightarrow b^{-}} \sqrt{2 \pi(b-x)} L_{1} / 2\left[\mathbf{f}_{1}^{\prime}(x)+\overline{\mathbf{f}_{1}^{\prime}(x)}\right] \\
& =\frac{2 \pi H^{-1}}{\sqrt{\pi b}} \sqrt{\frac{\frac{\pi b}{W} \sin \frac{\pi b}{W}}{\sin ^{2} \frac{\pi b}{2 W}-\sin ^{2} \frac{\pi a}{2 W}}} \operatorname{Re}\left\{-\mathbf{T} \sqrt{\frac{\sin ^{2} \frac{\pi z_{0}}{2 W}-\sin ^{2} \frac{\pi a}{2 W}}{\sin ^{2} \frac{\pi b}{2 W}-\sin ^{2} \frac{\pi z_{0}}{2 W}}}\right\} .
\end{aligned}
$$

Substituting (29) and (30) into (28) yields the corresponding ERRs. Furthermore, results (29) and (30) can serve as the Green's functions for finding the general IFs and ERRs of the edge-cracked piezoelectric strips under arbitrary electroelastic loadings in the strip.

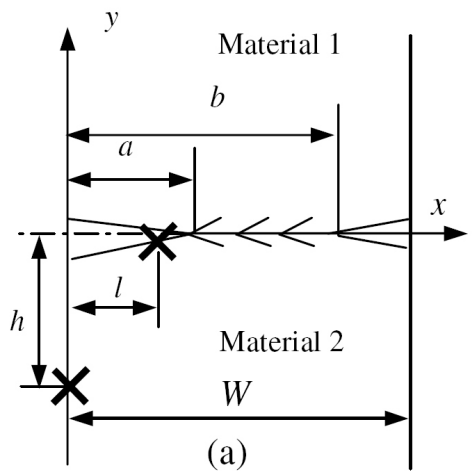

(a)

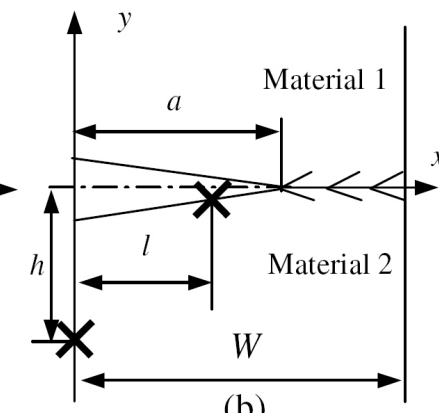

(b)

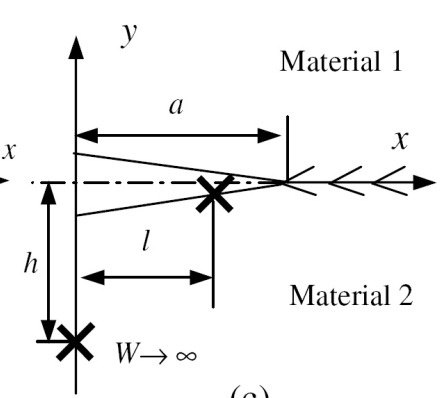

(c)

Figure 5. Cracked piezoelectric bimaterial with line-forces and line electric displacements on the crack surface and the free edge surface: (a) a twin-edge-cracked bimaterial strip, (b) an edge-cracked bimaterial strip, (c) an interfacial edge crack between two bonded quarter-planes. 


\section{Examples: strip free surface and crack surface with isolated line-forces and line-electric displacements}

Here we consider a special case as shown in Figure 5(a), in which two pairs of line-forces and lineelectric displacements are located at the crack surface and the left free-edge surface of the lower strip, respectively. Using definition (14), assume these two pairs of point electromechanical loadings, $\left(P_{1}, Q_{1}\right)$ and $\left(P_{2}, Q_{2}\right)$, are located at $z_{1}=l-0 \mathrm{i}$ and at $z_{2}=-\mathrm{i} h$ such that

$$
\mathbf{T}_{1}=\frac{L_{2}^{-1}}{2 \pi \mathrm{i}}\left[\begin{array}{c}
P_{1} \\
-Q_{1}
\end{array}\right], \text { at } z_{1}=l-0 \mathrm{i}, \quad \mathbf{T}_{2}=\frac{L_{2}^{-1}}{2 \pi \mathrm{i}}\left[\begin{array}{c}
P_{2} \\
-Q_{2}
\end{array}\right], \text { at } z_{2}=-\mathrm{i} h .
$$

Substitution of (31) into (29) and (30) yields

$$
\mathbf{K}^{a}=\frac{H^{-1} L_{2}^{-1}}{\sqrt{\pi a}} \sqrt{\frac{\frac{\pi a}{W} \sin \frac{\pi a}{W}}{\sin ^{2} \frac{\pi b}{2 W}-\sin ^{2} \frac{\pi a}{2 W}}}\left(\sqrt{\frac{\sin ^{2} \frac{\pi b}{2 W}-\sin ^{2} \frac{\pi l}{2 W}}{\sin ^{2} \frac{\pi a}{2 W}-\sin ^{2} \frac{\pi l}{2 W}}}\left[\begin{array}{c}
-P_{1} \\
Q_{1}
\end{array}\right]+\sqrt{\frac{\sin ^{2} \frac{\pi b}{2 W}+\sinh ^{2} \frac{\pi h}{2 W}}{\sin ^{2} \frac{\pi a}{2 W}+\sinh ^{2} \frac{\pi h}{2 W}}}\left[\begin{array}{c}
-P_{2} \\
Q_{2}
\end{array}\right]\right),
$$

and

$$
\mathbf{K}^{b}=\frac{H^{-1} L_{2}^{-1}}{\sqrt{\pi b}} \sqrt{\frac{\frac{\pi b}{W} \sin \frac{\pi b}{W}}{\sin ^{2} \frac{\pi b}{2 W}-\sin ^{2} \frac{\pi a}{2 W}}}\left(\sqrt{\frac{\sin ^{2} \frac{\pi a}{2 W}-\sin ^{2} \frac{\pi l}{2 W}}{\sin ^{2} \frac{\pi b}{2 W}-\sin ^{2} \frac{\pi l}{2 W}}}\left[\begin{array}{c}
-P_{1} \\
Q_{1}
\end{array}\right]+\sqrt{\frac{\sin ^{2} \frac{\pi a}{2 W}+\sinh ^{2} \frac{\pi h}{2 W}}{\sin ^{2} \frac{\pi b}{2 W}+\sin ^{2} \frac{\pi h}{2 W}}}\left[\begin{array}{c}
-P_{2} \\
Q_{2}
\end{array}\right]\right) .
$$

As a check, by letting $P_{1}=Q_{1}=0$ and $h \rightarrow \infty$, (32) and (33) reduce to

$$
\mathbf{K}^{a}=\frac{H^{-1} L_{2}^{-1}}{W} \sqrt{\frac{W \sin \frac{\pi a}{W}}{\sin ^{2} \frac{\pi b}{2 W}-\sin ^{2} \frac{\pi a}{2 W}}}\left[\begin{array}{c}
-P_{2} \\
Q_{2}
\end{array}\right], \quad \mathbf{K}^{b}=\frac{H^{-1} L_{2}^{-1}}{W} \sqrt{\frac{W \sin \frac{\pi b}{W}}{\sin ^{2} \frac{\pi b}{2 W}-\sin ^{2} \frac{\pi a}{2 W}}}\left[\begin{array}{c}
-P_{2} \\
Q_{2}
\end{array}\right] .
$$

which are the IFs of the edge-cracked strip with constant antiplane force $P_{2} / W$ and constant electric displacement $Q_{2} / W$ acting at the infinite end of the lower strip. The reduced homogenous case of result (34) covers those given by Li and Tang (2002), where a difference $1 / 2$ exists due to solution (34) only concerning the electromechanical loadings acting at the infinity of the lower strip.

Now let us consider some limiting geometry cases of (29). By letting $b \rightarrow W$ as shown in Figure $5(b)$, we obtain the IFs for a screw dislocation in an edge-cracked piezoelectric bimaterial strip as

$$
\mathbf{K}=4 \pi H^{-1} / \sqrt{\pi a} \sec \frac{\pi a}{2 W} \operatorname{Re}\left\{-\mathrm{i} \mathbf{T}\left[\frac{\pi a}{2 W} \operatorname{tg} \frac{\pi a}{2 W} /\left(\operatorname{tg}^{2} \frac{\pi a}{2 W}-\operatorname{tg}^{2} \frac{\pi z_{0}}{2 W}\right)\right]^{1 / 2}\right\} .
$$

Furthermore, by letting $W \rightarrow \infty$ (35) cover the IFs for an interfacial edge crack in a piezoelectric bimaterial half-plane as shown in Figure 5(c) as

$$
\mathbf{K}=4 \pi H^{-1} / \sqrt{\pi a} \operatorname{Re}\left(-\mathrm{i} \mathbf{T} a / \sqrt{a^{2}-z_{0}^{2}}\right) .
$$

When isolated electromechanical loadings are applied as (31), result (36) reduces to

$$
\mathbf{K}=2 \sqrt{a / \pi} H^{-1} L_{2}^{-1}\left(\frac{1}{\sqrt{a^{2}-l^{2}}}\left[\begin{array}{c}
-P_{1} \\
Q_{1}
\end{array}\right]+\frac{1}{\sqrt{a^{2}+h^{2}}}\left[\begin{array}{c}
-P_{2} \\
Q_{2}
\end{array}\right]\right),
$$

which accords to the solution given by Li and Fan (2001) and Wu et al. (2003).

The ERR of each crack configuration discussed above can be easily obtained by substituting (29)(30) and (32)-(37) into (28).

Since the general electroelastic potentials for the aforementioned edge-cracked piezoelectric bimaterial strips have been obtained explicitly, the whole electroelastic fields and the forces acting on the screw dislocation can be extracted straightforwardly using the method discussed by Pak (1990). 


\section{Concluding remarks}

Closed-form solutions are obtained for the electroelastic potentials and the fracture parameters of a screw dislocation in a piezoelectric bimaterial strip with twin interfacial edge cracks. Conformal mapping technique has been shown to be a powerful tool in solving some interfacial edge cracks in piezoelectric strips. The explicit solutions (29) and (30) can be employed as a useful theoretical base for the assessment of numerical analysis, especially for estimating the effect of the ratios $a / W$ and $b /$ $W$ on the IFs and ERRs in bimaterial piezoelectric strips. Furthermore, solutions (29) and (30) can be utilized as Green's functions to find the IFs and ERRs of the piezoelectric bimaterial strips under arbitrary antiplane mechanical and inplane electric loadings acting on the crack surfaces or in the strip out of the crack lines.

\section{Acknowledgments}

The authors would like to thank the anonymous reviewers for their helpful suggestions to improve this paper. Partial support of this work by the US Air Force Office of Scientific Research and the US Army Research Office is gratefully acknowledged.

\section{References}

Chen et al., 2002a - B. J. Chen, Z. M. Xiao, and K. M. Liew, On the interaction between a semi-infinite anti-crack and a screw dislocation in piezoelectric solid. Int. J. Solids Struct. 39 (2002), pp. 1505-1513.

Chen et al., 2002b - B. J. Chen, Z. M. Xiao, and K. M. Liew, A screw dislocation in a piezoelectric bi-material wedge. Int. J. Eng. Sci. 40 (2002), pp. 1665-1685.

Deeg, 1980 - Deeg, W. F., 1980. The analysis of dislocation, crack, and inclusion in piezoelectric solids. Ph.D. thesis, Stanford University, Stanford, CA

Kwon and Lee, 2002 - J. H. Kwon and K. Y. Lee, Electromechanical effects of a screw dislocation around a finite crack in a piezoelectric material. ASME J. Appl. Mech. 69 (2002), pp. 55-62.

Lee et al., 2000 - K. Y. Lee, W. G. Lee and Y. E. Pak, Interaction between a semi-infinite crack and a screw dislocation in a piezoelectric material. ASME J. Appl. Mech. 67 (2000), pp. 165-170.

Li, 2002 - X.-F. Li, Electroelastic analysis of an anti-plane shear crack in a piezoelectric ceramic strip. Int. J. Solids Struct. 39 (2002), pp. 1097-1117.

Li and Duan, $2001-$ X.-F. Li and X.-Y. Duan, The interaction of a screw dislocation and a free boundary in a piezoelectric material. Phys. Stat. Sol. Part B 227 (2001), pp. 613-619.

Li and Fan, 2001 - X.-F. Li and T.-Y. Fan, Mode-III interface edge crack between bonded quarter-planes of dissimilar piezoelectric materials. Arch. Appl. Mech. 71 (2001), pp. 703-714.

Li and Tang, 2002 - X.-F. Li and G.-J. Tang, Antiplane permeable edge cracks in a piezoelectric strip of finite width. Int. J. Fract. 115 (2002), pp. L35-L40.

Liu et al., 1999 - J.-X. Liu, S.-Y. Du, and B. Wang, A screw dislocation interacting with a piezoelectric bimaterial interface. Mech. Res. Commun. 26 (1999), pp. 415-420.

Pak, 1990 - Y. E. Pak, Force on a piezoelectric screw dislocation. ASME J. Appl. Mech. 57 (1990), pp. 863-869.

Parton, 1976 - V. Z. Parton, Fracture mechanics of piezoelectric materials. Acta Astronaut. 3 (1976), pp. 671-683.

Soh et al., 2002 - A. K. Soh, J.-X. Liu, and D.-N. Fang, A screw dislocation interacting with an interfacial crack in two dissimilar piezoelectric media. Phys. Stat. Sol. Part B 232 (2002), pp. 273-282.

Suo et al., 1992 - Z. Suo, C.-M. Kuo, D. M. Barnett, and J. R. Willis, Fracture mechanics for piezoelectric ceramics. J. Mech. Phys. Solids 40 (1992), pp. 739-765.

Wu et al., 2002 - X.-F. Wu, Y. A. Dzenis, and W.-S. Zou, Screw dislocation interacting with an interfacial edge crack between two bonded dissimilar piezoelectric wedges. Int. J. Fract. 117 (2002), pp. L9-L14.

Wu et al., 2003 - X.-F. Wu, S. Cohn, and Y. A. Dzenis, Screw dislocation interacting with interface and interfacial cracks in piezoelectric bimaterials. Int. J. Eng. Sci. 41 (2003), pp. 667-682.

Zhang et al., 2002 - T.-Y. Zhang, M. Zhao, and P. Tong, Fracture of piezoelectric ceramics. Adv. Appl. Mech. 38 (2002), pp. 147-289. 